\title{
Quantitative SEM/EDS, Step 1: What Constitutes a Sufficiently Flat Specimen?
}

\author{
Dale E. Newbury and Nicholas W. M. Ritchie \\ National Institute of Standards and Technology, Gaithersburg, MD 20899-8370
}

The most rigorous quantitative electron-excited $\mathrm{x}$-ray microanalysis that can be performed with scanning electron microscopy/energy dispersive $\mathrm{x}$-ray spectrometry (SEM/EDS) follows the k ratio protocol: measure the $\mathrm{x}$-ray intensity ratio $\mathrm{k}=\mathrm{I}_{\text {unknown }} / \mathrm{I}_{\text {standard }}$ for each element and calculate concentrations by applying matrix corrections for electron backscattering and energy loss (Z), $\mathrm{x}$-ray absorption (A), and characteristic- and continuum-induced secondary $\mathrm{x}$-ray fluorescence (F), as originally developed for the electron probe microanalyzer (EPMA) with wavelength dispersive spectrometry (WDS) [1]. By utilizing the k ratio protocol, SEM/EDS has been shown to be capable of matching the precision and accuracy of EPMA/WDS for major (concentration $\mathrm{C}>0.1$ mass fraction), minor $(0.01 \leq \mathrm{C} \leq 0.1)$, and upper trace range $(0.001<\mathrm{C}<0.01)$ constituents, even when significant peak interference occurs [2]. However, achieving the best analytical performance in practice with SEM/EDS or EPMA/WDS depends on measuring a properly prepared specimen that fulfills an often unstated requirement, namely that the only reason the measured x-ray intensities vary between the unknown and the standard(s) is that the composition is different. Geometric effects due to surface roughness can impact $\mathrm{x}$-ray generation and propagation sufficiently to violate this basic requirement, resulting in relative error contributions of a factor as high as 10 in severe cases [3]. How smooth must a surface be to reduce such geometric effects to an acceptable level? In the early development of the $\mathrm{k}$ ratio protocol, Yakowitz interrupted the metallographic grinding and polishing procedure at various stages to examine the variance in the measured $x$-ray intensities due to surface roughness for pure gold (comparing AuM $\alpha$ vs. AuL $\alpha$ ) and for a homogeneous brass alloy (comparing $\mathrm{CuK} \alpha$ vs. $\mathrm{ZnK} \alpha$ ) [4]. That study revealed that polishing to the practical limit then achievable with available polishing compounds was necessary to reduce the variance contribution due to surface roughness to an acceptable level for the case of "soft" compared to "hard" radiation, e.g., AuMa (2.12 keV) vs. AuLa, $(9.71 \mathrm{keV})$. What level of surface roughness creates significant geometric effects for the much lower energy x-rays of light elements, such as $\mathrm{O} \mathrm{K}=0.523 \mathrm{keV}$ ? The NIST Monte Carlo simulation embedded in DTSA-II has been used to examine this question [5]. A crenellated surface was modeled with the spacing and depth parameters of the grooves as variables, as shown schematically in Figure 1 with the EDS detector oriented to look across the grooves. Figure 2 shows the k ratios for the low energy photons $\mathrm{O} \mathrm{K}(0.523 \mathrm{keV})$ and FeL $(0.704$ $\mathrm{keV}$ ) in $\mathrm{FeO}$ (wustite) as a function of the spacing and depth of crenellations, revealing that geometric effects persist at the level of 5\% relative or greater down to crenellations with $100 \mathrm{~nm}$ height and lateral spacing. Polishing to a relief of less than $20 \mathrm{~nm}$ is needed to reduce the effects of surface roughness to a relative error contribution below $1 \%$ for these low energy photons.

[1] J. Goldstein et al., Scanning Electron Microscopy and X-ray Microanalysis, $3^{\text {rd }}$ ed. (Springer, New York, 2003) 391.

[2] N. Ritchie, D. Newbury, and J. Davis, Microsc. Microanal., 18 (2012) 892.

[3] D. Newbury and N. Ritchie, SPIE Proceedings, 8036 (2011) 803601-1.

[4] H.Yakowitz, in Fifty Years of Progress in Metallographic Techniques, ASTM SP430 (1968) 383.

[5] N. Ritchie, DTSA-II available free at: www.cstl.nist.gov/div837/837.02/epq/dtsa2/index.html 


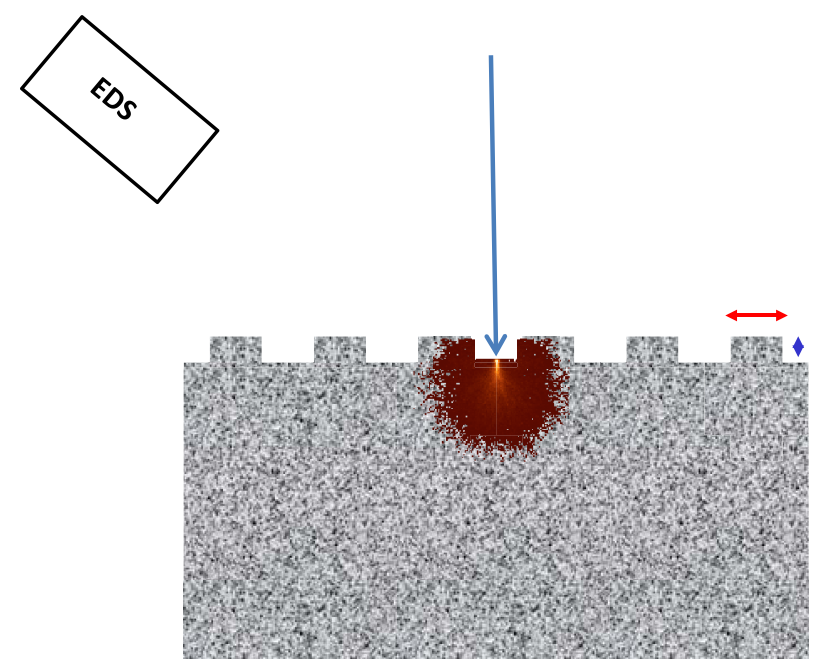

Figure 1. Schematic of DTSA-II NIST Monte Carlo simulation of a crenellated surface. The depth and spacing of the crenellations can be selected independently.

$$
\mathrm{FeO} \mathrm{E}_{0}=10 \mathrm{keV}
$$

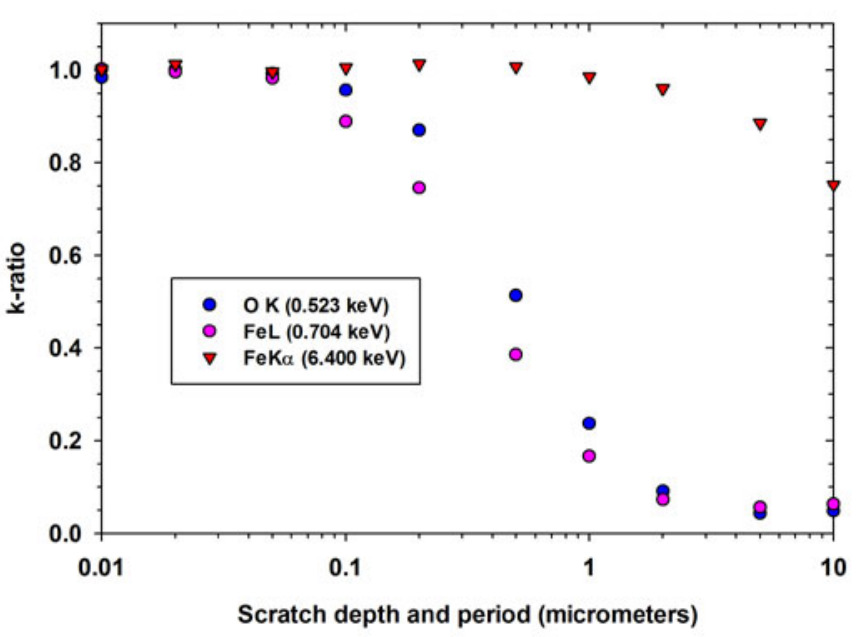

Figure 2. DTSA-II NIST Monte Carlo simulation of a crenellated surface of FeO with an incident beam energy of $E_{0}=10 \mathrm{keV}$. Note the severe decrease in $\mathrm{O} K$ and FeL intensity with increasing surface roughness. 